\title{
BRINGING ORDER TO HIGHER ORDER MOTOR DISORDERS
}

Maja Kojović, MD, $\mathrm{PhD}^{{ }^{*}}$ and Kailash P. Bhatia, MD, FRCP, ${ }^{2}$

Correspondence to:

Assoc. Prof. Maja Kojović, MD, PhD

Department of Neurology, University Medical Centre Ljubljana

Zaloška 2,1000 Ljubljana

Slovenia

Phone: +38641910533

maja.kojovic@kclj.si

Professor Kailash P Bhatia

Institute of Neurology

University College London

7 Queen Square

WC1N 3BG London

Tel: 08451555000

k.bhatia@ucl.ac.uk 


\section{ABSTRACT:}

Majority of movements in everyday situations are complex and involve volition, planning of the movement and selection of the motor program, all occurring before movement execution. Higher order motor disorders may be defined as abnormal motor behaviours resulting from disruption of any of the cortical processes that precede execution of the motor act. They are common in patients with neurodegenerative disorders, psychiatric diseases and structural brain lesions. These abnormal behaviours may be overlooked in the clinic, unless specifically evoked by the examiner. We discuss clinical and pathophysiological aspects of higher order motor disorders, including: (i) disorders of disinhibition, such as grasp reflex and grasping behaviour, utilisation and imitation behaviour, motor preservations and paratonia (ii) disorders of motor intention such as motor neglect and motor impersistence (iii) alien limb syndrome and (iv) motor overflow phenomena, such as mirror movements and synkinesias. A video illustration of each phenomenon is provided. We place the findings from recent neurophysiological studies within the framework of theories of motor control in order to provide better insight into pathophysiology of different disorders.

\section{INTRODUCTION}

Almost all movements performed in everyday situations are complex and involve volition, planning of the movement and selection of the motor programs for movement execution, while attentional and motivational factors interact to determine the goal of action. Higher order motor disorders may be understood as any abnormal motor behaviour that results from disruption of the cortical processes that precede execution of the motor action. Their pathophysiology has been poorly understood, but recent insights from neuroimaging studies and studies using transcranial magnetic stimulation (TMS), have helped our understanding of various aspects of cognitive motor control.

Although different higher order motor disorders have been clinically extensively studied by famous neurologist such as Lhermitte, Luria and Denny-Brown [1-4], their examination is 
usually not a part of standard neurological workup. Therefore, these abnormal behaviours may escape clinical detection.

In the present article, we discuss functional anatomy and clinical characteristics of higher order motor disorders and provide patients' videos to illustrate the phenomenology. We place the findings from recent neurophysiological studies within the framework of motor control theories in order to provide pathophysiological insight into different disorders. Included are (i) disorders of disinhibition;(ii) disorders of motor intention such as motor neglect and motor impersistence; (iii) alien limb syndromes and (iv) mirror movements . Apraxias, psychogenic motor disorders and disorders of motivated behaviour ( abulia and apathy) will not be considered, even though they may be viewed as a part of the spectrum of higher order motor disorders.

\section{DISINHIBITED BEHAVIORS}

Movement control, depending on whether the action is internally or externally guided, is mediated by two main motor circuits that converge on primary motor cortex (M1)[5].These are preSMA-M1 and parieto-premotor-M1 circuits. PreSMA-M1 circuit will dominate when the task is internally driven, as in self-paced finger movements or when gesturing without visual guidance or cues. Pre SMA is a part of supplementary motor area in the medial premotor cortex. It receives main inputs from prefrontal cortex and basal ganglia[6] and is considered a key structure for voluntary motor control [7]. With its connections with cognitive prefrontal brain and executive part of the motor circuit, preSMA is in strategic position to transform thoughts into action. Neuroimaging studies confirm stronger activation of preSMA with self-initiated movements compared to externally driven movements [8]. Electrical stimulation of pre SMA with subthresold intensities induces a feeling of an urge to move [9]. On the other hand, parieto-premotor-M1 circuit is involved when the motor actions depend on external cues[10] , i.e. when actions are driven by sensory stimuli. Within this network, information from primary sensory cortices is transferred to posterior parietal cortex, and then to the lateral part of premotor cortex, which in turn connects to M1. Extensive animal literature, as well as evidence in humans suggests that posterior parietal cortex has a role in creating multiple space representation to 
seize or handle objects under visual control[11-13], with parietal-premotor -M1 circuit being involved in object- oriented actions such as grasping[7]. PreSMA-M1 and parieto-premotorM1 circuits are normally balanced, so that we perform most movements without thinking on what we have to do, but without unintentionally touching or grasping objects from surroundings.

Precise functional anatomy of interactions between the two motor circuits remains unknown. Clinical evidence suggest that stimulus-driven actions transmitted by parietalpremotor circuit are inhibited by preSMA, because lesions of the preSMA result in automatic execution of actions in response to environmental triggers [14]. Lhermitte [1] hypothesised that with frontal lesions, inhibitory control of frontal over the parietal lobes is diminished, resulting in a release of the functions of the parietal lobes. Disbalance between preSMA-M1 and parieto-premotor-M1 networks in favour of later results in disinhibited behaviour, with affected person being dependent on visual and tactile stimulation from the outside world, so called environmental dependency syndrome [1, 2].

Frontal lobe signs may be easily overlooked in the clinic, unless they are specifically evoked by examiner. One of the reasons is that these patients might be most impaired in the situations of minimal external control. The outpatient clinic or hospital setting may impose sufficient external structure to suppress these behavioural tendencies [15].

Grasping is automatic tendency to grip objects. It is a stereotyped response consisting of forced hand closure on the object, provoked by distal ascending pressure on the palm (Video 1). It was first described by Denny-Brown and Chambers [3]. Interestingly, grasp reflex usually affects both hands even with unilateral lesions, with no hemispheric preferences. Grasping is not necessarily a forced phenomenon, as it may be temporally modified by will, when patient is instructed not to grasp, but will reappear as soon as attention is diverted. It may wax and wane from day to day, or even in the course of the same day. Grasping reflects the pathology of the frontal lobes, particularly medial frontal areas and cingulated gyrus or lesions of the basal ganglia [16]. Prehension (grasping) behaviour is more elaborated phenomenon. Here, patient's hand follows an object presented as tactile or visual stimuli in order to seize it (Video 2). Further, patients may engage in meaningless, repetitive and 
stereotypic actions, such as rubbing or manipulating clothes (e.g., buttons, collar) or bedclothes. Again, it is bilateral clinical phenomenon, even when the lesion is unilateral.

Utilisation behaviour is automatic tendency to manipulate surrounding objects [2]. Patients use the objects in appropriate way, but in an inappropriate situation ( Video 3). When challenged, patient will provide superficial explanation for such behaviour, such as "you told me to do it" or "i thought $i$ had to do it". Lhermitte's seminal description of utilisation behaviour includes a 52 year-old female patient with left frontal lobotomy. While in Professor Lhermitte's office, patient used blood pressure gauge to measure his blood pressure, the tongue depressor to examine his throat and the reflex hammer to test his ankle jerks. On another occasion the same patient obtained needle and syringe and gave professor Lhermitte gluteal injection[1].

Imitation behaviour may be regarded as dependence on social environment in the same sense as utilisation behaviour reflects dependence on physical environment [1]. Patient imitates the gestures and behaviour of the others, i.e. scratching the head or tapping the leg with hand in various rhythms. Criticism may be preserved, as patient may not imitate certain behaviours that are considered inappropriate. For these reason, there may be a distinction between imitation behaviour and echopraxia and echolalia, which are uncritical imitation of behaviour or speech, respectively. Imitation behaviour is seen in frontal lobe and basal ganglia lesions, Gilles de la Tourette syndrome, autistic spectrum disorders and in schizophrenia, suggesting it is an unspecific sign of impaired brain function[17] .

Imitation is main form of learning during development, which allows children to acquire many skills without the time-consuming process of trial-and-error learning. It is the persistence or re-emergence of imitation after a certain age that implies neurological or psychiatric disease. For example, persistence of echophenomena beyond the age of three may prompt the diagnostic consideration of autism spectrum disorders [17].

Current understanding of involuntary imitation phenomena must involve mirror neurons[18]. These are groups of nerve cells located in the posterior part of inferior frontal cortex and the anterior part of the inferior parietal lobule, interconnected into mirror neuronal system (MNS) . MNS receives visual input [19] and gets activated when monkey ( 
and human ) observes actions of others[20]. Observed acts are coded in the mirror areas, without normally triggering imitation, because MNS is suppressed by other areas of the frontal lobe . Imitation behaviour will arise as a consequence of lesion or dysfunction of areas that normally inhibit MNS. Indeed, the MNS in healthy adults may be under constant top-down inhibition by the anterior frontomedian cortex, the temporoparietal junction and frontoopercular cortex $[21,22]$.

Motor perseverations are inappropriate repetitions of the prior stimulus. Two types may be differentiated $[4,23,24]$. Recurrent perseverations (Figure 1) are inability to switch from one action to another, e.g. patient is asked to draw a circle and he performs correctly, but when further asked to draw triangle, he continues to draw circles. Continuous

perseverations are further repetition of an act although it should have been completed. An example is applause sign, typically seen in progressive supranuclear palsy and other neurodegenerative diseases with frontal lobe involvement $[25,26]$. Subject is asked to clap three times after demonstration by the examiner. The sign is positive if subject claps more than 3 times ( Video 4). Recurrent preservations are attributed to mesial frontal lobe lesions, while continuous preservations have been linked to dorsolateral prefrontal pathology [4].

Paratonia is involuntary resistance to passive movement due to apparent subject's inability to relax. The impression is that patient is actively resisting a passive movement. It can be demonstrated as the patient's limb remains elevated after being released by examiner, even after repeated instructions to relax (Video 5). The resistance of paratonia is variable and depends on the speed of passive movement and is proportional amount of force applied [27]. Slow movement provoke low resistance, while fast movements provoke high resistance. The resistance is the same in any direction and there is no clasp-knife phenomenon. Paratonia was first mentioned in 1910. by the French physicians Dupré, who noticed its connection with mental illness[28]. Kleist later described a phenomenon of very high muscle tone, which reacted against speeding up the movement during physical examination, in patients with dementia and he called it "gegenhalten" ("hold against") [29] 
. Occurrence and severity of paratonia increase with disease progression, from $10 \%$ in early to $90 \%$ in late stages of dementia, causing contractures and bedridden state in the terminal phase [30]. The nature of paratonia may change with dementia progression. Initially, patient may actively assist the passive movement by moving the limb in the same direction as the examiner, so called active assistance ("Mitgehen") or facilitatory paratonia ( Video 6) [31, 32]. Prevalence of paratonia in healthy eldery is approximately $1 \%$ [33], thus paratonia is almost always sign of brain disease. Paratonia is not exclusively related to Alzheimer's disease, but occurs in other forms of dementia [34]. It should be kept in mind that paratonia is involuntary increase of muscle tone and repeatedly instructing the patient to relax will not be helpful and may even worsen the paratonia. Pathophysiology of paratonia is unclear; anatomically it has been linked to frontal lobe dysfunction.

\section{DISORDERS OF INTENTION: MOTOR NEGLECT AND MOTOR IMPERSISTENCE}

Healthy people can make almost any kind of movement at any time because cognitive programs continuously provide the motor system with instructions how and when to move. According to Heilman [35]. , there are two major types of cognitive-motor programs, the praxis programs and the intentional programs. The praxis programs are "how" programs, providing spatial, temporal and force instructions for action. Intentional programs are "when" programs providing instructions, such as whether and when to move, when to stop moving and when to persist at an action. In right-handed people, disorders of the praxis, i.e. apraxias are almost always associated with left hemisphere dysfunction [36], while intentional disorders have right hemispheric preference [37]. Intentional disorders include motor neglect and motor impersistence and its variants.

Motor neglect is defined as under-utilization of a limb contralateral to brain lesion that cannot be fully explained by primary sensory and motor deficits (Video 7). Patients with motor neglect are unaware of their deficit [38] , but when reinforced by the examiner will improve strength and movement of the neglected limb. Motor neglect is twice more likely to affect patients suffering from right compared to left hemispheric stroke [39] and may have a 
significant impact on patient's performance and recovery. Spontaneous improvement of neglect occurs in proportion of patients, mostly in first 12- 14 weeks post-stroke [39-41] . There is relatively little information on how patients with motor neglect manage their everyday life, but increased determination of the patient, including the use of verbal strategies may result in tasks eventually being performed [42].

A milder form of motor neglect is motor extinction. Here, patient is able to better use contralesional limb in isolation, but the use of the same limb is impaired in bimanual tasks ( i.e. bottle opening). This may be seen on bedside examination as a deterioration in the performance of the affected limb ( hypometria, bradykinesis) during bimanual movements (Video 8).

The key characteristic of motor neglect is that motor deficit may be at least partially overcome by visual, sensory or auditory stimulation by the examiner or when the patient directs his attention and volition toward the affected limb $[41,42]$. These observations suggest that in motor neglect, habitual movements (movements performed in automatic fashion) might be more affected than goal directed movements. However, the opposite pattern of dissociation may be observed in patients with right SMA lesion[43] , as exemplified by one of our patients who was not able to move her left leg on command, while she could walk almost normally (Video 9).

Positron emission tomography (PET) and transcranial magnetic stimulation (TMS) studies suggest that primary motor cortex in motor neglect is intact, while the problem lays in the damage to the motor association areas and their connections with primary motor cortex. PET study in patients with motor neglect demonstrated normal metabolism in primary areas of motor output system (the primary sensorimotor cortex, basal ganglia, and cerebellum), while there was a hypometabolism in premotor, prefrontal, parietal, cingulate cortex[44]. Classen et al. suggested that motor neglect may be caused by enhanced cortical inhibition in the presence of intact corticospinal tract, presumably due to affection of afferent connections to primary motor cortex [45]. In TMS study, patients with motor 
neglect had normal motor evoked potential amplitudes and latencies, reflecting relatively intact corticospinal excitability, but at the same time cortical silent period was increased, reflecting increased GABA intracortical inhibition[45].

Motor impersistence is an inability to sustain motor act (movement or posture), unless repeatedly prompted by examiner. The symptom was first described by Fisher[46] in patients with right hemispheric stroke. Typical examples of motor impersistence include inability to keep eyes closed, tongue protruded, arm extended, inability to maintain conjugate gaze steadily in a fixed direction (Video 10) or inability to keep sustained arm grip , so called milkmaid sign". Motor impersistence is wildly appreciated among neurologists as a sign of Huntington's disease [47], but it has been also described in PSP [48] and in $10 \%$ of patients with $A D$, always in moderate or severe dementia[49]. Motor impersistence is observed in bihemispheric or diffuse brain diseases, however right frontal lesions seem to be particularly responsible [50]. In acute stroke, motor impersistence is more common in right comparing to left hemispheric lesions ( $23 \%$ vs. $9 \%$ ), further supporting right hemispheric dominance for motor persistence[51]. Callosal lesions that disconnect the left hemisphere from the right hemisphere inputs have also been associated with impersistence of the right limbs $[52,53]$. In stroke patients, similarly to motor neglect, impersistence may be transitory, present only in the acute phase. It is not know if motor impersistence affects patients day to day functioning. For example, gaze impersistence may interfere with ophthalmological workup, due to patient's inability to fixate the gaze. Motor impersistence should not be mistaken for uncooperativeness during rehabilitation [54]. Simultanapraxia is a subtype of motor impersistence, characterised by inability to perform simultaneous simple actions, such as closing the eyes and protruding the tongue at the same time.

\section{ALIEN LIMB PHENOMENA}

Alien limb refers to involuntary limb activity, in which patient reports loss of control over the affected limb or believes that the limb has been estranged. Doody and Jankovic [55] define alien limb as an involuntary motor activity together with a feeling that moving extremity is either "foreign" or has "a will of its own", in order to emphasise that foreignness is not consistently present in all alien hand variants [56]. The first description of alien hand 
syndrome (AHS), by Goldstein in 1908, was in a female who grabbed her own throat with the left hand and felt the hand had a will of its own [57].Current consensus in the literature is that there are three main variants of AHS: frontal, callosal (together called anterior variants) and posterior variant. Common to all variants is lack of sense of agency, i.e. loss of subjective awareness of initiating, executing, and controlling one's own volitional actions[58] . Lack of sense of ownership (limb foreignness) is however not universal. Patients experience it with the posterior variant, which us explained by coexistent hemineglect. Feeling of a limb as foreign is frequently absent in the frontal variant where patient recognize the limb as his own but acknowledge loss of its control.

Pure variants of AHS were initially described in patient with well defined lesions, such as stroke or tumour. When pathological process is widespread, as in neurodegenerative diseases, it is often difficult to observe isolated phenomenology, as there is overlap with other motor and cognitive deficits. For example, patient with advanced corticobasal degenration may not be able to acknowledge limb foreignness due to speech and language problems.

\section{Frontal alien hand}

The frontal form most commonly affects the dominant hand and is characterized by impulsive groping and compulsive manipulation of objects, with difficulties releasing grasped objects (Video 11). Frontal form of AHS has been called magnetic apraxia by Denny -Brown $[59,60]$. In frontal variant of alien limb, patient is aware that the arm belongs to him but is unable to control movements, as if the limb has "a will of its own". The common causes are neurodegenerative disorders with prominent frontal involvement and rarely stroke in the anterior communicating artery territory [57]. Explanation for frontal AHS is that pathology affecting medial frontal system, particularly preSMA-M1 circuit leaves the contralateral hand dependant on external stimuli operating through parieto-premotor circuit [61]. Lesion is usually in the dominant (left) medial frontal lobe (medial prefrontal cortex, supplementary motor area). This dominant hemisphere preference is explained by higher chance to develop motor neglect with non-dominant medial frontal lesions, which may mask frontal release behaviour [62]. 


\section{Callosal alien hand}

The callosal form of AHS is rare and tends to affect the non-dominant hand. The main abnormal behaviour is intermanual conflict. In an attempt to perform a task, the hands work inadequately together, with nondominant hand interrupting the dominant hand [63]. Activities such as tying shoelaces, buttoning shirt, using a knife and fork become difficult or impossible, which may be accompanied by an extreme sense of annoyance, anger and sometimes severe depression [64].

Callosal AHS may be caused by isolated corpus callosum injury due to surgical resection, callosal hemorrhage, infarction or callosal demyelination [65]. Mixed frontal and callosal AHS are due to extensive lesions of mesial frontal cortex extending to corpus callosum [62] and show combination of grasping behaviour and intermanual conflict. Pathophysiology of intermanual conflict is difficult to understand. Brainin et al. [64] hypothesised that implementation of any motor action includes simultaneous activation of inhibitory patterns in the opposite hemisphere. These inhibitory motor programs are normally suppressed by the processes of interhemispheric inhibition, i.e. inhibition from hemisphere preparing for voluntary movement toward opposite hemisphere. If interhemispheric inhibition fails due to pathology of corpus callosum, the conflicting motor programs may escape control.

\section{Posterior alien hand}

The posterior variant of AHS more commonly affects the non-dominant hand[66] and the lesion is typically in the right parietal lobe. Posterior AHS is associated with strong feelings of estrangement from the affected limb ("alien limb"), but less complex motor activity, which is manifested as limb levitation (Video 12) or non-purposeful and non-conflicting limb movements ( Video 13). Abnormal movements probably arise due to loss of proprioceptive feedback necessary for movement control, while feeling of limb being "alien" is due to coexisting neglect of the same limb[67]. Common aetiologies are neurodegeneration of the parieto-occipital cortex in corticobasal syndrome (corticobasal degeneration, CreutzfeldtJakob disease, Alzheimer's dementia) and stroke in the parietal lobe or posterior cerebral artery territory. Alien limb may rarely affect the leg ( Video 14) [68] . 
Another phenomenon from the spectrum of parietal AHS is hand avoidance behaviour [69]. Here, hand manifest withdrawing behaviour when stroked on the palmar surface, quite the opposite pattern from frontal alien hand. Denny-Brown suggested that parietal lobes are normally mediating approaching behaviour, with lesions causing the reverse- avoidance behaviour [70].

\section{Arm levitation}

In corticobasal syndrome, there may be a clinical continuity between spontaneous arm levitation, alien limb syndrome and useless hand. Spontaneous arm levitation appears particularly when attention is driven away from the limb (i.e., during walking, conversation or with eyes closed) and is not accompanied by denial of limb ownership. Patient is not even aware that arm is assuming abnormal posture and is able to bring it down on command. Disorder probably results from loss of proprioception arising from parietal lobe involvement. Arm levitation is sometimes seen in progressive suprenuclear palsy syndrome [71].

\section{Useless hand}

With progression of corticobasal syndrome, various combinations of apraxia, dystonia, rigidity, alien hand and motor neglect may lead to syndrome of useless hand. Affected arm is held in abnormal posture and patient does not use it, although there is no paresis, as evident by preserved stereotyped non-purposeful movements. Hand may still be used in automatic actions, for example as a support when standing up (Video 15).

\section{MOTOR OVERFLOW PHENOMENA: MIRROR MOVEMENTS AND SYNKINESIAS}

Motor overflow refers to involuntary movement that appear on voluntary movement of different body part. Mirror movements (MM) are involuntary movements of opposite (mirror) muscles that arise during voluntary movements of contralateral body region[72]. Motor mirroring is fascinating phenomenon because it reveals normal tendency of motor system to move in symmetrical fashion. On attempt to move one side only, symmetrical contralateral movements are normally suppressed by interhemispheric inhibition, which 
restrict motor output to the contralateral primary motor cortex (M1). MM reflect a failure of complex inhibitory neural circuits responsible for movement lateralization [73].

Alternative mechanism for MM generation , particularly relevant for cerebral palsy, is that motor output of voluntarily active M1 is transmitted ipsilaterally, either by uncrossed fastconducting corticospinal tract or by branching of crossed corticospinal fibers at the spinal level[74].

MM may be physiological (in children up to the age of six or in adults when induced under forceful or effortful conditions), congenital (rare genetic conditions) or acquired $[75,76]$. Acquired MM are seen cerebral palsy[74], particularly when uninjured hemisphere control movements of both sides (Video 16). Of interest for adult neurologist, MM are present in asymmetric neurodegenerative diseases such as Parkinson's disease (Video 17), corticobasal degeneration [73] (Video 18), but also in Huntington disease [77], motor neuron disease [78], essential tremor [79] and Friedreich ataxia [80]. As mirroring accompanies various neurological diseases, as well as schizophrenia [81], MM may be considered as an unspecific, soft neurological sign, that nevertheless points toward deficient brain inhibition. MM mainly involve distal upper limb muscles, sometimes causing difficulty in activities of daily living that require simultaneous but incongruent movements in each hand, such as typing on a keyboard, using fork and knife. More commonly, MM are noticed by attentive neurologist, whereas patients are not even aware of the symptom. Rarely, MM may be seen in proximal joints (Video 19) or in the leg and foot [82].

TMS is suitable to study MM. Using different TMS techniques, contralateral and ipsilateral corticospinal tract, as well as interhemispheric inhibition may be investigated, so that contribution of specific pathophysiological mechanism for MM generation in different diseases may be elucidated ( Figure 2). Considering aetiological diversity of MM, it is not surprising that pathophysiology of this phenomenon vary across different pathological conditions[83].

Synkinesias are subset of motor overflow whereby voluntary movements of one part of the body are accompanied by involuntary activation of other, non-mirroring muscles. For example, voluntary hand movement may be accompanied by involuntary movements of the 
ipsilateral leg or both legs (Video 20). Since the degree of motor maps overlap increase upstream of the primary motor cortex, synkinesias are likely to arise from dysfunction of the secondary motor areas, such as premotor cortex, SMA and cingulate and their connections to $\mathrm{M} 1[84-88]$.

$\mathrm{MM}$ and synkinesias should be differentiated from overflow phenomena in dystonia, that is spread of dystonia into the "unaffected" muscles, on activation of the dystonic limb [89]. Dystonia overfolow may be ipsilateral , for example ipsilateral shoulder elevation during writing in patient with graphospasm or contralateral, when on activation of the symptomatic arm dystonic movement/postures appears in the opposite, clinically unaffected arm. Term mirror dystonia has been used to denote dystonic postures and movements of the dominant ( affected) hand while patient with graphospasm writes with the nondominant ( non-affected) hand[90, 91]. Mirror dystonia may be useful to distinguish dystonic from secondary compensatory movements, when choosing appropriate muscles for botulinum toxin injections. Overflow phenomena in dystonia are explained by the existence of generalised neurophysiological dysfunction even when clinically dystonia is confined in one region. In writer's cramp, loss of intracortical inhibition may be found even in the hemisphere contralateral to the clinically unaffected side of the body[92] .

\section{CONCLUSIONS}

Higher order motor disorders are "hidden gem" of the brain, providing insight into interaction between motor and cognitive brain networks. These abnormal behaviours reflect difficulties in generating willed movements or failure of brain inhibitory functions, revealing complex top-down modulation of cognitive over motor networks. Investigation of higher order motor disorders starts from detailed clinical examination and structural neuroimaging. Functional imaging techniques may give further information on pathophysiology, by comparing patterns of activation in affected patients with healthy subjects performing comparable tasks. TMS is suitable to study various aspects of higher order motor disorders. Examples are processes of interhemispheric inhibition, relevant in MM and AHS ; functional state of corticospinal tract and intracortical inhibition, relevant in motor neglect; or parieto- 
frontal connectivity, relevant in disorders of disinhibition. In healthy people, TMS may be used to induce virtual lesion that may mimic higher order motor disorder [93].

\section{LEGENDS TO FIGURES:}

Figure 1. Recurrent motor perseverations. Patient with progressive supranuclear palsy was asked to write the name of his country: Slovenia. He gets stuck and repeats writing letter S, until prompted to write letter L. He then preserve writing letter L.

Figure 2. Use of TMS in studying mirror movements. Patient with cerebral palsy with right hemiparesis, caused by stroke at age 2 (Video 16). Focal TMS stimulation of the affected ( left) hemisphere (b) did not elicit any contralateral (or ipsilateral) motor evoked potentials (MEP) in the EMG. Focal stimulation of unaffected hemisphere (a) produced MEP responses of short and similar latency in both contralateral and the ipsilateral muscles. In the contralateral muscles, the mean latency of MEP is $20.4 \mathrm{~ms}$ in first dorsal interossus muscle (FDI) and $21.4 \mathrm{~ms}$ in abductor pollicis brevis muscle (APB), while the ipsilateral latency is $20.5 \mathrm{~ms}$ and $20.3 \mathrm{~ms}$, respectively. These finding suggest that unaffected hemisphere control movements of both affected and unaffected side, which explains why MM appear on voluntary movements of either side. Abbreviations: Rt, right hemisphere; Lt, left hemisphere; M1, primary motor cortex; MSO, maximal stimulator output

\section{VIDEO LEGENDS:}

Video 1. Grasping reflex. Grasping is present on both side and consists of forced hand closure over the hammer's handle. After instructed not to grasp, patient resisted it, showing grasping is not absolutely forced phenomenon.

Video 2: Prehension( grasping) behaviour. Patient with corticobasal syndrome follows with hands an object presented as tactile and visual stimulus, in an attempt to seize it. This behaviour is spontaneous and occurs while examiner is talking to patient's spouse. The patient did not get any instructions.

Video3. Utilisation behaviour. Various objects were put in front of the patient with frontal lobe tumour, without giving him any instructions. The patient first uses neurological 
hammer, he then put on the gloves on and finally starts eating cookies. When doctors asked him why he put the gloves on, he had no answer.

Video 4. Motor preservations (continuous). Patient with progressive supranuclear palsy and positive applause sign. He was asked to clap three times after demonstration by the examiner, however he clasped many times.

Video 5. Paratonia. Despite repeated suggestion by examiner, the patient is not able to relax during examination of the muscle tone. Limb stay lifted up in the air after quick release by examiner.

Video 6. Facilitatory paratonia. Patient with early Alzheimer dementia actively assist the passive movements. When the arm is released, it becomes obvious that patient is making the same active movements as were the passive movements made by examiner.

Video 7. Motor neglect. The patient has right frontoparietal metastasis . It can be noticed that he shaved only the right half of the face. On instruction to lift the arms up, he lifts only the right arm. On explicit instruction to lift both arms up, he is able to do it. On instruction to put arms down, he release only the right arm , while he still keep holding the handle with his left arm. Finally, he puts the left arm down after directly prompted by examiner.

Video 8. Motor extinction. During bimanual movements, the performance of the left, affected limb, is deteriorating until it finally stops moving.

Video 9. Motor neglect in right SMA lesion. The patient underwent surgery for right SMA cortical dysplasia (as treatment for pharmaco- resistant epilepsy) and suffered postoperative oedema. Few days following surgery, she could not move the left leg on command (both supine and when sitting), although she could walk almost normally. Patient was completely aware of the problem, which remitted after 2 weeks.

Video 10. Gaze impersistence. Patient is unable to keep eyes fixated to the target, particularly on leftward gaze, with eyes constantly drifting toward midline. When looking up, eyes drift down, in ping-pong fashion. 
Video 11. Frontal alien hand syndrome. Patient with corticobasal syndrome magnetically follows an object with dominant hand only and finally forcefully grasp it.

Video 12. Patient with parietal lobe stroke complained his right arm was spontaneously lifting and hee felt it as not being his arm. Right arm spontaneously slowly levitates (both with eyes open and closed).

Video 13. Alien hand in corticobasal syndrome. Left arm manifesst non-purposeful movements, while right arm is held in abnormal posture and patient does not utilise it ( useless hand).

Video 14. Alien leg. Patient with corticobasal syndrome manifest spontaneous levitation of the left leg that may last for hours. She has patellar dislocation due to these prolonged abnormal postures.

Video 15. Useless hand. Left arm is held in an abnormal flexed posture and the patient does not use it, apart for stereotyped nose pocking. The patient also use his left arm as a support when standing up, suggesting that automatic movements are preserved.

Video 16. The patient with cerebral palsy and right hemiparesis shows mirror movements when moving both, affected and non-affected arm( see also Figure 2).

Video 17. Parient with Parkinson's disease shows mirror movements when finger tapping with the more affected (right side), while no mirror movements are seen when moving less affected side.

Video 18. Mirror movements in patient with corticobasal syndrome. On attempt to imitate meaningless gestures with right (more affected side), MM are seen on the opposite side.

Video 19. Mirror movements in proximal joints. Mirroring appears in the right elbow joint, when the patient performs finger to nose test with left arm.

Video 20 . When the patient intentionally open and close the left fist, synkinesias ( synchronous involuntary movements of the same direction and speed) appear in both legs. 


\section{ETHICAL STANDARDS}

The manuscript does not contain clinical studies. All patients gave their informed consent for video recording and publication in clinical/scientific journal.

CONFLICT OF INTEREST STATEMENT: The authors declare that they have no conflict of interest.

\section{REFERENCES:}

1. Lhermitte, F., Human autonomy and the frontal lobes. Part II: Patient behavior in complex and social situations: the "environmental dependency syndrome". Ann Neurol, 1986. 19(4): p. 335-43.

2. Lhermitte, F., 'Utilization behaviour' and its relation to lesions of the frontal lobes. Brain, 1983. 106 (Pt 2): p. 237-55.

3. Denny-Brown, D. and R.A. Chambers, The parietal lobe and behavior. Res Publ Assoc Res Nerv Ment Dis, 1958. 36: p. 35-117.

4. Luria, A.R., Two Kinds of Motor Perseveration in Massive Injury of the Frontal Lobes. Brain, 1965. 88: p. 1-10.

5. Goldberg, G., Supplementary motor area structure and function: Review and hypotheses. Behavioral and Brain Sciences 17:187-245, 1985.

6. Strick, P.L., R.P. Dum, and N. Picard, Motor areas on the medial wall of the hemisphere. Novartis Found Symp, 1998. 218: p. 64-75; discussion 75-80, 104-8.

7. Haggard, P., Human volition: towards a neuroscience of will. Nat Rev Neurosci, 2008. 9(12): p. 934-46.

8. Deiber, M.P., et al., Mesial motor areas in self-initiated versus externally triggered movements examined with fMRI: effect of movement type and rate. J Neurophysiol, 1999. 81(6): p. 3065-77.

9. Fried, I., et al., Functional organization of human supplementary motor cortex studied by electrical stimulation. J Neurosci, 1991. 11(11): p. 3656-66.

10. Passingham, R.E., Two cortical systems for directing movement. Ciba Found Symp, 1987. 132: p. 151-64.

11. Mountcastle, V.B., et al., Posterior parietal association cortex of the monkey: command functions for operations within extrapersonal space. J Neurophysiol, 1975. 38(4): p. 871-908.

12. Taira, M., et al., Parietal cortex neurons of the monkey related to the visual guidance of hand movement. Exp Brain Res, 1990. 83(1): p. 29-36.

13. Jeannerod, M., et al., Grasping objects: the cortical mechanisms of visuomotor transformation. Trends Neurosci, 1995. 18(7): p. 314-20.

14. Pacherie, E., The anarchic hand syndrome and utilization behavior: a window onto agentive self-awareness. Funct Neurol, 2007. 22(4): p. 211-7.

15. Mesulam, M.M., Frontal cortex and behavior. Ann Neurol, 1986. 19(4): p. 320-5.

16. De Renzi, E. and C. Barbieri, The incidence of the grasp reflex following hemispheric lesion and its relation to frontal damage. Brain, 1992. 115 Pt 1: p. 293-313. 
17. Ganos, C., et al., The pathophysiology of echopraxia/echolalia: relevance to Gilles de la Tourette syndrome. Mov Disord, 2012. 27(10): p. 1222-9.

18. Rizzolatti, G., et al., Premotor cortex and the recognition of motor actions. Brain Res Cogn Brain Res, 1996. 3(2): p. 131-41.

19. Allison, T., A. Puce, and G. McCarthy, Social perception from visual cues: role of the STS region. Trends Cogn Sci, 2000. 4(7): p. 267-278.

20. Rizzolatti, G. and L. Craighero, The mirror-neuron system. Annu Rev Neurosci, 2004. 27: p. 169-92.

21. Brass, M., J. Derrfuss, and D.Y. von Cramon, The inhibition of imitative and overlearned responses: a functional double dissociation. Neuropsychologia, 2005. 43(1): p. 89-98.

22. Bien, N., et al., The brain's intention to imitate: the neurobiology of intentional versus automatic imitation. Cereb Cortex, 2009. 19(10): p. 2338-51.

23. Sandson, J. and M.L. Albert, Varieties of perseveration. Neuropsychologia, 1984. 22(6): p. 715-32.

24. Goldberg, E., Varieties of perseveration: a comparison of two taxonomies. J Clin Exp Neuropsychol, 1986. 8(6): p. 710-26.

25. Wu, L.J., et al., Applause sign in Parkinsonian disorders and Huntington's disease. Mov Disord, 2008. 23(16): p. 2307-11.

26. Luzzi, S., et al., Applause sign: is it really specific for Parkinsonian disorders? Evidence from cortical dementias. J Neurol Neurosurg Psychiatry, 2011. 82(8): p. 830-3.

27. Hobbelen, J.S., et al., Paratonia: a Delphi procedure for consensus definition. J Geriatr Phys Ther, 2006. 29(2): p. 50-6.

28. Dupre, E., Débilité mentale et débilité motrice associées. Rev Neurol. , 1910. 20: p. 5456.

29. Kleist, K., Gegenhalten (motorischer negativismus) zwangsgreifen und thalamus opticus. Monatchr Psychiat Neurol, 1927. 65: p. 317-396.

30. Souren, L.E., E.H. Franssen, and B. Reisberg, Neuromotor changes in Alzheimer's disease: implications for patient care. J Geriatr Psychiatry Neurol, 1997. 10(3): p. 938.

31. Beversdorf, D.Q. and K.M. Heilman, Facilitory paratonia and frontal lobe functioning. Neurology, 1998. 51(4): p. 968-71.

32. Chatterjee, A., Feeling frontal dysfunction: facilitory paratonia and the regulation of motor behavior. Neurology, 1998. 51(4): p. 937-9.

33. Benassi, G., et al., Neurological examination in subjects over 65 years: an epidemiological survey. Neuroepidemiology, 1990. 9(1): p. 27-38.

34. Hobbelen, J.S., et al., Diagnosing paratonia in the demented elderly: reliability and validity of the Paratonia Assessment Instrument (PAI). Int Psychogeriatr, 2008. 20(4): p. 840-52.

35. Heilman, K.M., Intentional neglect. Front Biosci, 2004. 9: p. 694-705.

36. Donkervoort, M., et al., Prevalence of apraxia among patients with a first left hemisphere stroke in rehabilitation centres and nursing homes. Clin Rehabil, 2000. 14(2): p. 130-6.

37. Buxbaum, L.J., et al., Hemispatial neglect: Subtypes, neuroanatomy, and disability. Neurology, 2004. 62(5): p. 749-56. 
38. Garbarini, F., et al., Dissociations and similarities in motor intention and motor awareness: the case of anosognosia for hemiplegia and motor neglect. J Neurol Neurosurg Psychiatry, 2013. 84(4): p. 416-9.

39. Siekierka-Kleiser, E.M., et al., Quantitative assessment of recovery from motor hemineglect in acute stroke patients. Cerebrovasc Dis, 2006. 21(5-6): p. 307-14.

40. Nijboer, T.C., B.J. Kollen, and G. Kwakkel, Time course of visuospatial neglect early after stroke: a longitudinal cohort study. Cortex, 2013. 49(8): p. 2021-7.

41. Sampanis, D.S. and J. Riddoch, Motor neglect and future directions for research. Front Hum Neurosci, 2013. 7: p. 110.

42. Laplane, D. and J.D. Degos, Motor neglect. J Neurol Neurosurg Psychiatry, 1983. 46(2): p. 152-8.

43. Bannur, U. and V. Rajshekhar, Post operative supplementary motor area syndrome: clinical features and outcome. Br J Neurosurg, 2000. 14(3): p. 204-10.

44. von Giesen, H.J., et al., Cerebral network underlying unilateral motor neglect: evidence from positron emission tomography. J Neurol Sci, 1994. 125(1): p. 29-38.

45. Classen, J., et al., The motor syndrome associated with exaggerated inhibition within the primary motor cortex of patients with hemiparetic. Brain, 1997. 120 ( Pt 4): $p$. 605-19.

46. Fisher, M., Left hemiplegia and motor impresistence. J Nerv Ment Dis, 1956. 123(3): p. 201-18.

47. Walker, F.O., Huntington's disease. Lancet, 2007. 369(9557): p. 218-28.

48. Cambier, J., et al., [Frontal syndrome of progressive supranuclear palsy]. Rev Neurol (Paris), 1985. 141(8-9): p. 528-36.

49. Lopez, O.L., J.T. Becker, and F. Boller, Motor impersistence in Alzheimer's disease. Cortex, 1991. 27(1): p. 93-9.

50. Kertesz, A., et al., Motor impersistence: a right-hemisphere syndrome. Neurology, 1985. 35(5): p. 662-6.

51. De Renzi, E., M. Gentilini, and C. Bazolli, Eyelid movement disorders and motor impersistence in acute hemisphere disease. Neurology, 1986. 36(3): p. 414-8.

52. Seo, S.W., et al., Dominant limb motor impersistence associated with callosal disconnection. Neurology, 2007. 68(11): p. 862-4.

53. Kim, H.J., et al., Callosal Motor Impersistence: A Novel Disconnection Syndrome. Cogn Behav Neurol, 2017. 30(2): p. 68-72.

54. Rosse, R.B. and C.P. Ciolino, Motor impersistence mistaken for uncooperativeness in a patient with right-brain damage. Psychosomatics, 1986. 27(7): p. 532-4.

55. Doody, R.S. and J. Jankovic, The alien hand and related signs. J Neurol Neurosurg Psychiatry, 1992. 55(9): p. 806-10.

56. Goldberg, G. and K.K. Bloom, The alien hand sign. Localization, lateralization and recovery. Am J Phys Med Rehabil, 1990. 69(5): p. 228-38.

57. Hassan, A. and K.A. Josephs, Alien Hand Syndrome. Curr Neurol Neurosci Rep, 2016. 16(8): p. 73.

58. Moore, J.W. and P.C. Fletcher, Sense of agency in health and disease: a review of cue integration approaches. Conscious Cogn, 2012. 21(1): p. 59-68.

59. Denny-Brown, D., The nature of apraxia. J Nerv Ment Dis, 1958. 126(1): p. 9-32. 
60. Peluso, S., et al., The magnetic apraxia of Denny-Brown. Neurology, 2016. 87(6): p. 644.

61. Boccardi, E., et al., Utilisation behaviour consequent to bilateral SMA softening. Cortex, 2002. 38(3): p. 289-308.

62. Chan, J.L. and E.D. Ross, Alien hand syndrome: influence of neglect on the clinical presentation of frontal and callosal variants. Cortex, 1997. 33(2): p. 287-99.

63. Sperry, R.W., Hemisphere deconnection and unity in conscious awareness. Am Psychol, 1968. 23(10): p. 723-33.

64. Brainin, M., A. Seiser, and K. Matz, The mirror world of motor inhibition: the alien hand syndrome in chronic stroke. J Neurol Neurosurg Psychiatry, 2008. 79(3): p. 24652.

65. Feinberg, T.E., et al., Two alien hand syndromes. Neurology, 1992. 42(1): p. 19-24.

66. Assal, F., S. Schwartz, and P. Vuilleumier, Moving with or without will: functional neural correlates of alien hand syndrome. Ann Neurol, 2007. 62(3): p. 301-6.

67. Graff-Radford, J., et al., The alien limb phenomenon. J Neurol, 2013. 260(7): p. 18808.

68. Chan, J.L., R.S. Chen, and K.K. Ng, Leg manifestation in alien hand syndrome. J Formos Med Assoc, 1996. 95(4): p. 342-6.

69. Fitzgerald, D.B., et al., Asymmetrical alien hands in corticobasal degeneration. Mov Disord, 2007. 22(4): p. 581-4.

70. D., D.-B., Positive and negative aspects of cerebral cortical function. N C Med J 1956 17: p. 295-303.

71. Barclay, C.L., C. Bergeron, and A.E. Lang, Arm levitation in progressive supranuclear palsy. Neurology, 1999. 52(4): p. 879-82.

72. Beaule, V., S. Tremblay, and H. Theoret, Interhemispheric control of unilateral movement. Neural Plast, 2012. 2012: p. 627816.

73. Cox, B.C., M. Cincotta, and A.J. Espay, Mirror movements in movement disorders: a review. Tremor Other Hyperkinet Mov (N Y), 2012. 2.

74. Farmer, S.F., et al., Plasticity of central motor pathways in children with hemiplegic cerebral palsy. Neurology, 1991. 41(9): p. 1505-10.

75. Addamo, P.K., et al., The effects of age and attention on motor overflow production-A review. Brain Res Rev, 2007. 54(1): p. 189-204.

76. Farmer, S.F., Mirror movements in neurology. J Neurol Neurosurg Psychiatry, 2005. 76(10): p. 1330.

77. Hashimoto, T., M. Shindo, and N. Yanagisawa, Enhanced associated movements in the contralateral limbs elicited by brisk voluntary contraction in choreic disorders. Clin Neurophysiol, 2001. 112(9): p. 1612-7.

78. Krampfl, K., et al., Mirror movements and ipsilateral motor evoked potentials in ALS. Amyotroph Lateral Scler Other Motor Neuron Disord, 2004. 5(3): p. 154-63.

79. Louis, E.D., E. Rios, and C. Henchcliffe, Mirror movements in patients with essential tremor. Mov Disord, 2009. 24(15): p. 2211-7.

80. Low, S.C., et al., Excessive motor overflow reveals abnormal inter-hemispheric connectivity in Friedreich ataxia. J Neurol, 2013. 260(7): p. 1757-64.

81. Hoy, K.E., et al., Neurological soft signs in schizophrenia: investigating motor overflow. World J Biol Psychiatry, 2009. 10(4 Pt 3): p. 763-71. 
82. Tubbs, R.S., et al., Exclusive lower extremity mirror movements and diastematomyelia. Pediatr Neurosurg, 2004. 40(3): p. 132-5.

83. Cincotta, M. and U. Ziemann, Neurophysiology of unimanual motor control and mirror movements. Clin Neurophysiol, 2008. 119(4): p. 744-62.

84. PENFIELD W, B.E., SOMATIC MOTOR AND SENSORY REPRESENTATION IN THE CEREBRAL CORTEX OF MAN AS STUDIED BY ELECTRICAL STIMULATION. Brain, 1937. 60(4): p. 389-443.

85. Schieber, M.H. and L.S. Hibbard, How somatotopic is the motor cortex hand area? Science, 1993. 261(5120): p. 489-92.

86. Sanes, J.N., et al., Shared neural substrates controlling hand movements in human motor cortex. Science, 1995. 268(5218): p. 1775-7.

87. Fink, G.R., et al., Multiple nonprimary motor areas in the human cortex. J Neurophysiol, 1997. 77(4): p. 2164-74.

88. Park, I.S., et al., Mirror movements and involuntary homolateral limb synkinesis in a patient with probable Creutzfeldt-Jakob disease. Clin Neurol Neurosurg, 2009. 111(4): p. 380-3.

89. Sitburana, O. and J. Jankovic, Focal hand dystonia, mirror dystonia and motor overflow. J Neurol Sci, 2008. 266(1-2): p. 31-3.

90. Jedynak, P.C., C. Tranchant, and D.Z. de Beyl, Prospective clinical study of writer's cramp. Mov Disord, 2001. 16(3): p. 494-9.

91. Espay, A.J., Motor excess during movement: Overflow, mirroring, and synkinesis. Clin Neurophysiol, 2010. 121(1): p. 5-6.

92. Ridding, M.C., et al., Changes in the balance between motor cortical excitation and inhibition in focal, task specific dystonia. J Neurol Neurosurg Psychiatry, 1995. 59(5): p. 493-8.

93. Ghacibeh, G.A., et al., Dissociation of neglect subtypes with transcranial magnetic stimulation. Neurology, 2007. 69(11): p. 1122-7. 